\title{
Do Electroweak Precision Data and Higgs-Mass Constraints Rule Out a Scalar Bottom Quark with Mass of Order $5 \mathrm{GeV}$ ?
}

\author{
M. Carena, ${ }^{1}$ S. Heinemeyer, ${ }^{2}$ C. E. M. Wagner, ${ }^{3}$ and G. Weiglein ${ }^{4}$ \\ ${ }^{1}$ Fermilab, Box 500, Batavia, Illinois 60510-0500 \\ ${ }^{2}$ HET, Physics Department, Brookhaven National Laboratory, Upton, New York 11973 \\ ${ }^{3}$ High Energy Division, Argonne National Laboratory, Argonne, Illinois 60439 \\ and the Enrico Fermi Institute, University of Chicago, 5640 Ellis, Chicago, Illinois 60637 \\ ${ }^{4}$ Theoretical Physics Division, CERN, CH-1211 Geneva 23, Switzerland \\ (Received 14 August 2000; revised manuscript received 3 November 2000)
}

\begin{abstract}
We study the implications of a scalar bottom quark, with a mass of $\mathcal{O}(5 \mathrm{GeV})$, within the minimal supersymmetric standard model. Light sbottoms may naturally appear for large $\tan \beta$ and, depending on the decay modes, may have escaped experimental detection. We show that a light sbottom cannot be ruled out by electroweak precision data and the bound on the lightest $C \mathcal{P}$-even Higgs-boson mass. We infer that a light $\tilde{b}$ scenario requires a relatively light scalar top quark whose mass is typically about the top-quark mass. In this scenario the lightest Higgs boson decays predominantly into $\tilde{b}$ pairs and obeys the mass bound $m_{h} \lesssim 123 \mathrm{GeV}$.
\end{abstract}

DOI: 10.1103/PhysRevLett.86.4463

PACS numbers: 12.60.Jv, 12.15.Lk, 14.80.Cp

New light particles, with masses of the order of the weak scale, are an essential ingredient in any scenario beyond the standard model (SM) that leads to an explanation of the large hierarchy between the Planck mass and the weak scale. Although no clear evidence of such a particle has been reported so far, searches for new particles are usually performed under model-dependent assumptions, and hence the quoted bounds may not be valid if these assumptions are relaxed. In particular, we shall investigate whether a light scalar bottom quark, $\tilde{b}$, with mass close to the bottomquark mass, $m_{b}$, is consistent with present experimental data [1]. A light $\tilde{b}$ is most naturally obtained within supersymmetric theories [2] for large values of $\tan \beta$, as required in minimal $\mathrm{SO}(10)$ scenarios [3]. Supersymmetric theories have received much attention in the last years since they provide an elegant way to break the electroweak symmetry and to stabilize the huge hierarchy between the grand unification and the Fermi scales; they also allow for a consistent unification of the gauge couplings. Supersymmetry (SUSY) predicts the existence of scalar partners to each SM fermion, and spin- $1 / 2$ partners to the gauge and Higgs bosons.

Scalar particles, like the $\tilde{b}$, have been searched for at current and past collider experiments. Despite being in the mass reach of these colliders, they may have been overlooked for several reasons. Bottom squarks give only a tiny contribution to the inclusive cross section for $e^{+} e^{-} \rightarrow$ hadrons, smaller than $2 \%$ of the total quark contribution for five flavors of quarks, and therefore small compared to the experimental error in these measurements [1]. Furthermore, due to a $p$-wave suppression of the fermion contribution to its decay width, a $\tilde{b} \tilde{\bar{b}}$ resonance would be difficult to extract from background [4]. Concerning the semileptonic decay of the $\tilde{b}, \tilde{b} \rightarrow c l+$ missing energy, if its branching ratio is small, for instance, of about the bottom quark one, the exclusion bound derived by the CLEO Collaboration does not apply [5]. If, on the other hand, the light $\tilde{b}$ decays into a light quark and missing energy, due to its small mass and the small mass splitting between the $\tilde{b}$ and its decay products, it cannot be detected through missing energy searches in $e^{+} e^{-}$or hadron colliders [1]. If, instead, the $\tilde{b}$ decays fully hadronically with no missing energy, it will remain undetected due to its small contribution to the hadronic cross section at hadron and lepton colliders. Finally, the presence of a light $\tilde{b}$ will slightly affect the extrapolated value of the electromagnetic and strong gauge couplings, $\alpha_{e m}$ and $\alpha_{s}$, at the scale $M_{Z}$ : the variation induced on $\alpha_{e m}\left(M_{Z}\right)$ is smaller than the difference between the two most commonly used values of $\alpha_{e m}\left(M_{Z}\right)$ [6]. The variations of both $\alpha_{e m}$ and $\alpha_{s}\left(M_{Z}\right)$ are smaller than the present error on the respective coupling [1].

On the other hand, the hadronic observables measured with high precision at the $Z$ peak at LEP1 [7] impose tight and fairly model-independent constraints on this kind of new physics, provided that the $\tilde{b}$ couples with sufficient strength to the $Z$. A necessary condition for such a scenario within the minimal supersymmetric standard model (MSSM) to be phenomenologically viable is thus a relatively small coupling of the $\tilde{b}$ to the $Z$ boson. The squark couplings to the $Z$ depend on the mixing angle, $\theta_{\tilde{q}}$,

$$
\begin{aligned}
& g_{Z \tilde{q}_{1} \tilde{q}_{1}} \simeq g\left(T_{3} \cos ^{2} \theta_{\tilde{q}}-Q_{\tilde{q}} \sin ^{2} \theta_{W}\right), \\
& g_{Z \tilde{q}_{1} \tilde{q}_{2}} \simeq g T_{3} \sin \theta_{\tilde{q}} \cos \theta_{\tilde{q}}, \\
& g_{Z \tilde{q}_{2} \tilde{q}_{2}} \simeq g\left(T_{3} \sin ^{2} \theta_{\tilde{q}}-Q_{\tilde{q}} \sin ^{2} \theta_{W}\right),
\end{aligned}
$$

where $\sin ^{2} \theta_{W} \equiv s_{W}^{2}=1-M_{W}^{2} / M_{Z}^{2}$; in the following the shorthand notation $s_{\tilde{q}} \equiv \sin \theta_{\tilde{q}}$ and $c_{\tilde{q}} \equiv \cos \theta_{\tilde{q}}$ is used. In the particular case of the $\tilde{b}, Q_{\tilde{b}}=-1 / 3, T_{3}=-1 / 2$, and hence an exact cancellation of the coupling of the lightest $\tilde{b}, \tilde{b}_{2}$, to the $Z$ is achieved in lowest order when 
$s_{\tilde{b}}^{2}=2 / 3 s_{W}^{2}$, i.e., $\left|s_{\tilde{b}}\right| \approx 0.38$. Similarly, an exact cancellation for the lightest $\tilde{t}, \tilde{t}_{1}$, yields $c_{\tilde{t}}^{2}=4 / 3 s_{W}^{2}$. For our conventions in the squark sector, see Ref. [8].

Besides the constraints from the direct search and from $Z$-peak observables for the $\tilde{b}$, the considerable splitting between the masses in the scalar bottom and top sector, which are necessary to avoid direct observation of at least one of these particles at LEP, gives rise to sensitive restrictions from virtual effects to electroweak precision observables, e.g., $\sin ^{2} \theta_{\text {eff }}, M_{W}, \Gamma_{l}$, via contributions to the $\rho$ parameter. Therefore, it is of interest to investigate whether a $\tilde{b}$ almost mass degenerate with the bottom quark is consistent with the strong constraints from electroweak precision data. A further crucial question is whether a light $\tilde{b}$ scenario can give rise to a sufficiently large value for the lightest $C \mathcal{P}$-even Higgs-boson mass in the MSSM in view of the bounds arising from the Higgs searches at LEP. The latter constraints have meanwhile ruled out a considerable part of the parameter space, even in the unconstrained MSSM (in which no assumptions about the underlying SUSY-breaking mechanism are made) [9]. The present bound on the SM Higgs mass from the direct search is $M_{H}>113.3 \mathrm{GeV}$ at $95 \%$ C.L. [10]. The upper bound on the lightest $C \mathcal{P}$-even Higgs mass within the MSSM is $m_{h} \lesssim 130 \mathrm{GeV}$ for $m_{t}=175 \mathrm{GeV}$. This bound arises from the theoretical prediction of $m_{h}$ in the MSSM up to the two-loop level $[8,11]$.

As a first step in our analysis we have calculated the production cross section for light scalar bottoms as a function of the effective $Z \tilde{b}_{2} \tilde{b}_{2}$ coupling (throughout this paper we use the tree-level notation for this coupling, although it can be viewed as an effective coupling containing loop corrections). As an additional scenario to the case where this coupling precisely vanishes, we have taken the sbottom mixing in the range $\left|s_{\tilde{b}}\right| \approx 0.3-0.45$. If the $\tilde{b}$ would decay with a small semileptonic decay width, in a way similar to the bottom quark, it would mainly affect observables associated with bottom production, as discussed below. Analyzing the corresponding effects on the relevant $Z$ peak observables, $R_{b}, R_{c}, R_{l}, A_{F B}^{b}$, $A_{b}, \Gamma_{\text {had }}, \Gamma_{Z}$, and $\sigma_{\text {had }}$, for $\left|s_{\tilde{b}}\right|=0.3,0.45$ we find the following results for the comparison of the data with the predictions, given in units of standard deviations: $\delta R_{b}=$ $0.40 \sigma(1.0 \sigma), \delta R_{c}=-1.01 \sigma(-1.04 \sigma), \delta R_{l}=0.62 \times$ $\sigma(1.08 \sigma), \delta A_{F B}^{b}=-2.33 \sigma(-2.42 \sigma), \delta A_{b}=-0.48 \times$ $\sigma(-0.55 \sigma), \quad \delta \Gamma_{\text {had }}=0.09 \sigma(0.57 \sigma), \quad \delta \Gamma_{Z}=$ $-0.85 \sigma(-0.43 \sigma), \delta \sigma_{\text {had }}=1.87 \sigma(1.62 \sigma)$. The values in brackets correspond to the SM predictions [7]. The agreement of the predictions with the data improves over the SM case for most observables. Lowering $\alpha_{s}$ by $\approx 0.0018(\approx 0.6 \sigma)[1], R_{l}, \Gamma_{\text {had }}, \Gamma_{Z}$, and $\sigma_{\text {had }}$ would reach their SM values, whereas $R_{b}, R_{c}, A_{F B}^{b}$, and $A_{b}$ would to a good approximation keep the above improved values. Thus, a small but nonvanishing coupling of the light $\tilde{b}$ to the $Z$ not only is compatible with the hadronic observables at the $Z$ peak, but may even slightly improve the agreement with the data. Since the shifts discussed here are small, the overall quality of a global fit to all data is expected to change only slightly. The same is true if, alternatively, the sbottom decays only hadronically.

As a second step in our analysis, we investigate the constraints from $\Delta \rho$ and the Higgs mass limit for the following two cases.

(I) Vanishing coupling of $\tilde{b}_{2}$ and $\tilde{t}_{1}$ to the $Z$ boson, $s_{\tilde{b}}= \pm \sqrt{2 / 3} s_{W}, c_{\tilde{t}}= \pm \sqrt{4 / 3} s_{W}$.

(II) Small $Z \tilde{b}_{2} \tilde{b}_{2}$ couplings corresponding to the range of mixing angles $\left|s_{\tilde{b}}\right| \approx 0.3-0.45$. No constraints on the $Z \tilde{t}_{1} \tilde{t}_{1}$ coupling are imposed.

In the analysis below, $m_{\tilde{b}_{2}}$ has been fixed to $4 \mathrm{GeV}$, but varying this mass by a few $\mathrm{GeV}$ would not qualitatively change our results. Since we also restrict $s_{\tilde{b}}$ as specified above, in principle there are four more free parameters left in the scalar bottom and top sector, $m_{\tilde{b}_{1}}, m_{\tilde{t}_{1}}, m_{\tilde{t}_{2}}$, and $s_{\tilde{t}}$. The relation between these parameters in the masseigenstate basis and the ones in the basis of the current eigenstates $\tilde{b}_{L}, \tilde{b}_{R}, \tilde{t}_{L}, \tilde{t}_{R}$ is given by the mixing matrices

$$
\mathcal{M}_{\tilde{q}}^{2}=\left(\begin{array}{cc}
M_{\tilde{q}_{L}}^{2}+m_{q}^{2}+D_{\tilde{q}_{L}} & m_{q} X_{q} \\
m_{q} X_{q} & M_{\tilde{q}_{R}}^{2}+m_{q}^{2}+D_{\tilde{q}_{R}}
\end{array}\right)
$$

for $q=t, b, \quad$ and $\quad X_{t}=A_{t}-\mu \cot \beta, \quad X_{b}=A_{b}-$ $\mu \tan \beta$. The $D$-term contributions $D_{\tilde{q}_{L, R}}$ have not explicitly been written. In the above, $A_{t, b}$ denote the trilinear Higgs- $\tilde{t}$, Higgs- $\tilde{b}$ couplings, respectively, and $\mu$ is the Higgs mixing parameter. $\mathrm{SU}(2)$ gauge invariance leads to the relation $M_{\tilde{t}_{L}}=M_{\tilde{b}_{L}}$. Thus only three of the four parameters $m_{\tilde{b}_{1}}, m_{\tilde{t}_{1}}, m_{\tilde{t}_{2}}, s_{\tilde{t}}$ are independent.

Since the heavier $\tilde{b}$ has not been observed at LEP2, and it can in principle be produced in association with the lighter one, its mass should be larger than (conservatively) $\sim 200 \mathrm{GeV}$. Neglecting terms of order $m_{\tilde{b}_{2}}^{2} / m_{\tilde{b}_{1}}^{2}$, the mass of the heavier $\tilde{b}$ is given as $m_{\tilde{b}_{1}}^{2}=m_{b} X_{b} /\left(s_{\tilde{b}} c \tilde{b}\right)$. In order to generate a sufficiently large value of $m_{\tilde{b}_{1}}$, relatively large values of $X_{b}$ are required. They can naturally be obtained for values of $|\mu|$ and $A_{b}$ around the squark masses if $\tan \beta \approx\left|s_{\tilde{b}} c_{\tilde{b}}\right| m_{\tilde{b}_{1}} / m_{b}$, where $m_{b} \approx 3 \mathrm{GeV}$ is the $\overline{\mathrm{MS}}$ running bottom mass at the weak scale. For heavy $\tilde{b}_{1}$ masses of order $400 \mathrm{GeV}$ and $\tilde{b}$ mixing angles of the cases (I) and (II), this implies values of $\tan \beta \gtrsim 30$.

Concerning the constraints from contributions of the $\tilde{t}-\tilde{b}$ sector to $\Delta \rho$, the present data leave some room for a small but nonzero contribution to $\Delta \rho$. We use $2 \times 10^{-3}$ as upper bound for SUSY contributions [1]. We have checked that a limit on $\Delta \rho^{\text {SUSY }}$ as tight as $3 \times 10^{-4}$ does not qualitatively change our results.

Regarding the Higgs mass constraints, beyond the tree level, the main correction to $m_{h}$ stems from the $t-\tilde{t}$ sector and, for large values of $\tan \beta$, also from the $b-\tilde{b}$ sector. For a light $\tilde{t}$ and $\tilde{b}$ sector, the Higgs tends to be light. For large values of $\tan \beta$ and $M_{A}$, however, the Higgs may be heavy enough to avoid LEP constraints, but tends naturally to be in the range $110-120 \mathrm{GeV}$. Concerning the bounds 
obtained at LEP2, one should note that the off-diagonal term in the $\tilde{b}$ mass matrix of the order of the square of the weak scale [i.e., a large value of $(\mu \tan \beta)$ ] results in a large coupling of these sbottoms to the lightest $C \mathcal{P}$-even Higgs boson. Therefore, for large $\tan \beta$ and $M_{A}$ the width of its decay into sbottoms,

$$
\Gamma\left(h \rightarrow \tilde{b}_{2} \tilde{\tilde{b}}_{2}\right) \sim G_{F} \sqrt{2}\left(m_{b} \mu \tan \beta s_{\tilde{b}} c_{\tilde{b}}\right)^{2} /\left(8 \pi m_{h}\right),
$$

will be much larger than the corresponding one into bottoms, $\Gamma(h \rightarrow b \bar{b}) \sim G_{F} \sqrt{2}\left(m_{h} m_{b}^{2}\right) /(4 \pi)$.

The limits from LEP will depend strongly on the decay modes of the sbottoms. As a conservative bound, we adopt the present lower bound on the Higgs boson of the $\mathrm{SM}$ at LEP2, $m_{h} \gtrsim 113.3 \mathrm{GeV}$ [10]. This is consistent with the assumption that the light $\tilde{b}$ decay channels are similar to the bottom quark ones. However, if it decayed fully hadronically with no missing energy or into down (or strange) quarks and missing energy, considerably weaker Higgs mass bounds would be obtained.

For the case of a very light $\tilde{b}$, with a non-negligible component on the left-handed $\tilde{b}$, the constraint from the $\rho$ parameter demands a relatively light $\tilde{t}$. The simultaneous requirement that the lightest $C \mathcal{P}$-even Higgs mass should be above the experimental bound leads to strong restrictions in the $\tilde{t}$ sector. In the numerical analysis, we use the following parameters: $m_{t}=174.3 \mathrm{GeV}, m_{b}=3 \mathrm{GeV}$, $\tan \beta=40, \quad M_{A}=800 \mathrm{GeV}, \quad m_{\tilde{g}}=200 \mathrm{GeV}, \quad \mu=$ $\pm 250 \mathrm{GeV}, M_{2}=200 \mathrm{GeV}$. We have chosen a large value for $M_{A}$, yielding that the upper bound for $m_{h}$ within this scenario is only weakly dependent on the actual value of this parameter [12]. The dependence on $m_{\tilde{g}}, \mu$, and $M_{2}$ is also weak.

The theoretical predictions for $m_{h}$ employed here are based on the two-loop results of Refs. [8,11,13], implemented in the programs FEYNHIGGS [14] and SUBHPOLE $[11,13]$. We have checked that the results for $m_{h}$ obtained with the two programs are close to each other and therefore lead to similar conclusions. $\Delta \rho^{\text {SUSY }}$, including leading two-loop contributions [15], has been evaluated with FEYNHIGGS.

The analysis is performed for the cases (I) and (II) defined above. It should be emphasized that, although case (I) seems highly constrained, starting from the requirement of a small $\tilde{b}_{2}$ mass and a vanishing coupling to the $Z$, and requiring the left-handed $\tilde{t}$ mass to be larger than the right-handed one, most solutions to the precision observables and Higgs mass constraints would lead to a small coupling of the lightest $\tilde{t}$ to the $Z$.

In Fig. 1 the allowed parameter regions for $m_{\tilde{t}_{1}}$ and $m_{\tilde{t}_{2}}$ for the cases (I) and (II) are shown, obeying the $m_{h}$ and $\Delta \rho^{\text {SUSY }}$ constraints. For both cases a considerable part of the parameter space is consistent with the constraints. In case (I) the allowed regions are $70 \mathrm{GeV} \lesssim$ $m_{\tilde{t}_{1}} \lesssim 220 \mathrm{GeV}, 450 \mathrm{GeV} \lesssim m_{\tilde{t}_{2}} \lesssim 600 \mathrm{GeV}$. In case (II) the $\tilde{t}$ masses obey the constraints for $70 \mathrm{GeV} \lesssim m_{\tilde{t}_{1}}$

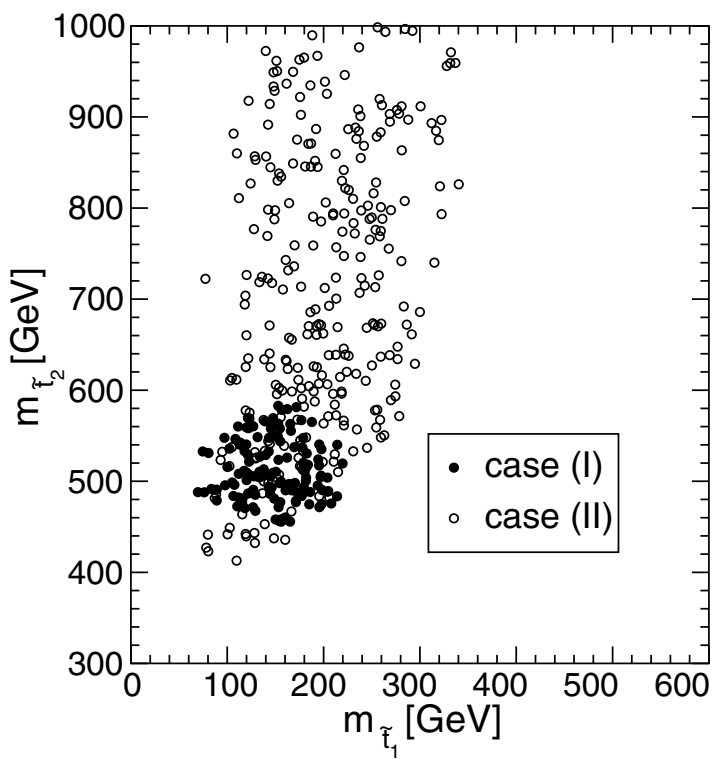

FIG. 1. Regions in the $m_{\tilde{t}_{1}}-m_{\tilde{t}_{2}}$ plane for the cases (I) and (II), allowed by the requirements $m_{h} \gtrsim 113.3 \mathrm{GeV}$ and $\Delta \rho^{\text {SUSY }}<$ 0.002. (See text for the other parameters.)

$\lesssim 330 \mathrm{GeV}, 400 \mathrm{GeV} \lesssim m_{\tilde{t}_{2}}$, and we considered values of $m_{\tilde{t}_{2}} \leq 1000 \mathrm{GeV}$.

In Fig. 2 the allowed parameter regions for $m_{h}$ are shown. In case (I) the lightest $C \mathcal{P}$-even Higgs will always be lighter than $120 \mathrm{GeV}$, while in case (II) slightly larger values of $m_{h}$ can be obtained, $m_{h} \lesssim 123 \mathrm{GeV}$. If the light sbottoms decay in a way similar to the $b$ quarks, this offers good chances for the Higgs boson discovery at the Tevatron or the LHC, using its associated production with the gauge bosons [16] or with the top quark $[17,18]$.

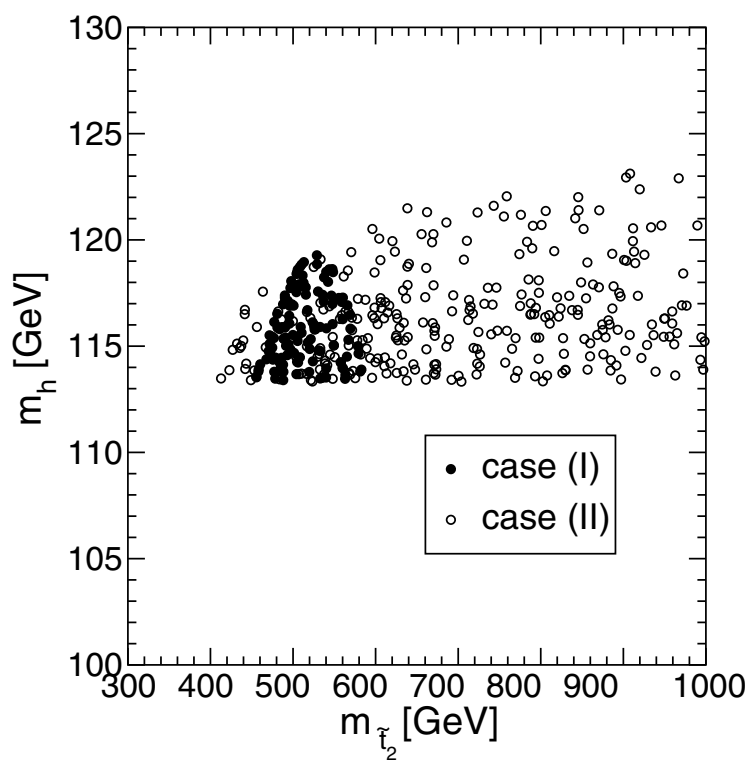

FIG. 2. Regions in the $m_{\tilde{t}_{2}}-m_{h}$ plane for the cases (I) and (II), allowed by the requirements $m_{h} \geqslant 113.3 \mathrm{GeV}$ and $\Delta \rho^{\text {SUSY }}<$ 0.002. (See text for the other parameters.) 
Scalar top masses below or about $100 \mathrm{GeV}$ are constrained by LEP data. This mainly applies to case (II), in which no constraints on the $Z \tilde{t}_{1} \tilde{t}_{1}$ coupling were imposed, therefore allowing for larger contributions from $Z$ exchange to the $\tilde{t}_{1} \tilde{t}_{1}$ production cross section. It follows from Figs. 1 and 2 that only small changes would be obtained if this bound were applied.

Let us stress that the fine-tuning of the parameters necessary to accommodate a $\tilde{b}$ mass of about $m_{b}$ is about the same as the one necessary to realize the SM with new physics arising at energies of about a few TeV. Concerning the mixing in the $\tilde{b}$ and $\tilde{t}$ sector, the analysis of case (II) shows that the mixing angles can be varied over a considerable range, e.g., $\left|s_{\tilde{b}}\right| \approx 0.3-0.45$, without leading to conflicts with the experimental constraints. The fact that not much fine-tuning is necessary is reflected in the large amount of experimentally consistent models (see Figs. 1 and 2).

In conclusion, a light $\tilde{b}$ within the MSSM cannot at present be ruled out by the electroweak precision data and the Higgs mass constraints from LEP2. Even in the most extreme case of vanishing couplings of the lightest $\tilde{t}$ and the lightest $\tilde{b}$ to the $Z$, an allowed parameter region within the MSSM is found, resulting in an upper value for $m_{h}$, $m_{h} \lesssim 120 \mathrm{GeV}$, for $m_{t}=174.3 \mathrm{GeV}$. If the light $\tilde{b} \mathrm{de}-$ cays like a $b$ quark and has a small but nonvanishing coupling to the $Z$ boson, this may even yield a slightly better agreement of the $Z$ peak observables with the experimental data than in the SM. In this case $m_{h}$ is restricted to be $m_{h} \lesssim 123 \mathrm{GeV}$. An important finding in both cases is that the scenario with a $\tilde{b}$ almost mass degenerate to the $b$ quark requires, in general, also a light $\tilde{t}$ whose mass is typically around the $t$ quark mass. If it is light enough, such a $\tilde{t}$ should be accessible at Run II of the Tevatron. If the sbottoms decay similarly to $b$ quarks, these light stops and sbottoms could contribute to the third-generation quark cross sections, whereas the measured Tevatron cross sections are, in general, larger than the SM expectations [19]. Besides promising very interesting phenomenological implications for Run II of the Tevatron and for the LHC, a scenario with a light $\tilde{b}$ could also be studied in detail at the upcoming $b$ factories.

G. W. thanks G. Buchalla, A. Dedes, H. Dreiner, M. L. Mangano, and D. Zeppenfeld for interesting discussions. S. H. thanks H. Eberl, S. Kraml, and C. Schappacher for technical support. C. W. and M. C. thank E. Berger, B. Dobrescu, B. Harris, D. Kaplan, H. Logan, J. Lykken,
S. Martin, K. Matchev, S. Mrenna, U. Nierste, M. Schmitt, Z. Sullivan, T. Tait, D. Wackeroth, and G. Wolf for stimulating discussions and comments. This work was supported in part by U.S. DOE, Division of HEP, Contract No. W-31-109-ENG-38.

[1] Particle Data Group, Eur. Phys. J. C 15, 1 (2000).

[2] H. E. Haber and G. Kane, Phys. Rep. 117, 75 (1985); H. P. Nilles, Phys. Rep. 110, 1 (1984).

[3] M. Olechowski and S. Pokorski, Phys. Lett. B 214, 393 (1988); B. Ananthanarayan, G. Lazarides, and Q. Shafi, Phys. Rev. D 44, 1613 (1991).

[4] C. R. Nappi, Phys. Rev. D 25, 84 (1982).

[5] CLEO Collaboration, V. Savinov et al., hep-ph/0010047.

[6] S. Eidelman and F. Jegerlehner, Z. Phys. C 67, 585 (1995); M. Davier and A. Höcker, Phys. Lett. B 435, 427 (1998).

[7] The LEP Collaborations, LEP EWWG and SLD, lepewwg.web.cern.ch/LEPEWWG/Welcome.html.

[8] S. Heinemeyer, W. Hollik, and G. Weiglein, Phys. Rev. D 58, 091701 (1998); Phys. Lett. B 440, 296 (1998); Eur. Phys. J. C 9, 343 (1999).

[9] P. Bock et al., Report No. CERN-EP/2000-055.

[10] A. Read, LEPC presentation, 20.07.2000, lephiggs.web.cern.ch/LEPHIGGS.

[11] M. Carena, M. Quirós, and C.E. M. Wagner, Nucl. Phys. B461, 407 (1996).

[12] M. Carena, S. Heinemeyer, C.E.M. Wagner, and G. Weiglein, hep-ph/9912223.

[13] M. Carena, H.E. Haber, S. Heinemeyer, W. Hollik, C. E. M. Wagner, and G. Weiglein, Nucl. Phys. B580, 29 (2000).

[14] S. Heinemeyer, W. Hollik, and G. Weiglein, Comput. Phys. Commun. 124, 76 (2000).

[15] A. Djouadi, P. Gambino, S. Heinemeyer, W. Hollik, C. Jünger, and G. Weiglein, Phys. Rev. Lett. 78, 3626 (1997); Phys. Rev. D 57, 4179 (1998).

[16] M. Carena, S. Mrenna, and C.E. M. Wagner, Phys. Rev. D 60, 075010 (1999); Phys. Rev. D 62, 055008 (2000); M. Carena, H. E. Haber, J. Conway, J. Hobbs (conveners), Higgs Working Group of the Tevatron Run II Workshop, hep-ph/0010338.

[17] J. Goldstein, C.S. Hill, J. Incandela, S. Parke, D. Rainwater, and D. Stuart, hep-ph/0006311.

[18] ATLAS Collaboration, Report No. CERN/LHCC/99-15 (1999).

[19] CDF Collaboration, F. Abe et al., Phys. Rev. Lett. 80, 2779 (1998); 71, 2400 (1993); D0 Collaboration, B. Abbott et al., Phys. Rev. Lett. 83, 1908 (1999); 84, 5478 (2000). 